\title{
Covariant derivative of product tensors
}

\author{
by Józef JoAChim Telega (Katowice)
}

Introduction. In paper [6] we have presented the axiomatic definition of the covariant derivative of $(H, \bar{H})$-product tensors. Now we shall deal with the special case where $H(A)=A, \bar{H}(B)=B$.

Making use of Theorem 2.1 from paper [6] we shall give in Section 1 formulae for the covariant derivatives of product tensors of valences $(1,1 ; 0,0),(0,0 ; 1,1)$ and $(1,0 ; 0,1)$, respectively. The case where manifolds have the same dimensions and there is a mapping between them has been dealt with in Section 2 . In the final remarks we shall show how our considerations imply the formula for the covariant derivative of connecting tensors given in [2].

1. First let us recall the definition of a product tensor. A geometric product object $t^{i a}$ is called a product tensor of valence $(1,1 ; 0,0)$ when its law of transformation has the form [3], [7],

$$
t^{i^{\prime a^{\prime}}}=A_{i}^{i^{\prime}} B_{a}^{a^{\prime}} t^{i a}, \quad i=1, \ldots, n, a=1, \ldots, \bar{n},
$$

where $A_{i}^{i^{\prime}}=\frac{\partial x^{i^{\prime}}}{\partial x^{i}}, B_{a}^{\alpha^{\prime}}=\frac{\partial y^{a^{\prime}}}{\partial y^{a}} ; x^{i}, y^{\alpha}$ denote the coordinates of points $x \in \bar{X}^{n}, y \in Y^{\bar{n}}$, respectively (in certain coordinate systems), and $x^{i^{*}}, y^{a^{\prime}}$ their coordinates in arbitrary other coordinate systems, $\dot{X}^{n}, Y^{\bar{n}}$ are manifolds of dimensions $n, \bar{n}$ respectively. Throughout this paper we adopt the following convention: Latin indices run from 1 to $n$, and Greek indices from $1, \ldots, \bar{n}$.

We conclude from Theorem 2.1 of paper [6] that the covariant derivative of the product tensor (1.1) has the form

where

$$
D_{i \beta}^{j a}=C_{i \beta}^{k} t_{j k}^{j \alpha}+D_{i \beta}^{\nu} t_{, \gamma}^{j a}+E_{i k \beta \gamma}^{j a} t^{k \nu}
$$

$$
t_{, k}^{j a}=\frac{\partial t^{j a}}{\partial x^{k}}, \quad t_{j \gamma}^{j a}=\frac{\partial t^{j a}}{\partial y^{\gamma}} .
$$

In this paper we shall donsider only the special case of (1.2), namely where (see Remark 2.1 of [6])

$$
E_{i k \beta \gamma}^{j a}=C_{\beta} G_{i k \gamma}^{j a}+D_{i} K_{k \beta \gamma}^{j a} .
$$


Further, we assume that the covariant derivative of the product scalar $\sigma(x, y)$ has the form (see Remark 1.1 of [6])

where

$$
f_{i a}=C_{a} \sigma_{, i}+D_{i} \sigma_{, a},
$$

$$
\sigma_{, i}=\frac{\partial \sigma(x, y)}{\partial x^{i}}, \quad \sigma_{, a}=\frac{\partial \sigma(x, y)}{\partial y^{a}}
$$

Then, as was pointed out in [6] for a more general case of the covariant derivative of the $(H, \bar{H})$-product tensors, we have

$$
D_{i \beta}^{j a}=C_{\beta}\left(t_{, i}^{j a}+G_{i k \gamma}^{j a} t^{k \gamma}\right)+D_{i}\left(t_{, \beta}^{j a}+K_{k \beta \gamma}^{j a} t^{k \gamma}\right) .
$$

Let us denote the expressions in brackets in the following way:

$$
\dot{\nabla}_{i} t^{j a}=t_{. i}^{j a}+G_{i k \beta}^{j a} t^{k \beta}, \quad \dot{\nabla}_{\beta} t^{j \alpha}=t_{, \beta}^{j a}+K_{k \beta \gamma}^{j a} t^{k \gamma} ;
$$

thus we obtain

$$
D_{i \beta}^{j a}=C_{\beta} \dot{\nabla}_{i} t^{j a}+D_{i} \dot{\nabla}_{\beta} t^{j a}
$$

We see that it is sufficient to consider only the covariant derivative $\dot{\nabla}_{i}$, because for the covariant derivative $\dot{\nabla}_{\beta}$ our subsequent considerations would be analogical.

Making use of Remark 2.2 of [6], we obtain the law of transformation of the product object $G_{i k \beta}^{j a}$ in the form

$$
G_{i^{\prime} k^{\prime} \beta^{\prime}}^{j^{\prime} a^{\prime}}=A_{j}^{j^{\prime}} A_{i^{\prime}}^{i} A_{k^{\prime}}^{k} B_{a}^{a^{\prime}} B_{\beta^{\prime}}^{\beta} G_{i k \beta}^{j a}-\delta_{\beta^{\prime}}^{a^{\prime}} A_{i^{\prime}}^{i} A_{k^{\prime}}^{k} A_{i k}^{j^{\prime}}-\delta_{k^{\prime}}^{j^{\prime}} A_{i^{\prime}}^{i} B_{\beta^{\prime}}^{a} B_{a i}^{a^{\prime}}
$$

where

$$
A_{k i}^{j^{\prime}}=\frac{\partial^{2} x^{j^{\prime}}}{\partial x^{i} \partial x^{k}}, \quad B_{a i}^{a^{\prime}}=\frac{\partial^{2} y^{a^{\prime}}}{\partial x^{i} \partial y^{a}}
$$

If a manifold $X^{n}$ is a space $L^{n}$, i.e. a space with a linear connexion $\Lambda_{i k}^{j}$, then we obtain the special form of relation $\left(1.4_{1}\right)$, putting

$$
\dot{\nabla}_{i} t^{j a} \stackrel{\mathrm{d} f}{=} t_{; i}^{j a}=t_{, i}^{j a}+\Lambda_{i k}^{j} t^{k a},
$$

where the semicolon denotes the covariant derivative of single tensors (defined at a point). Thus we get

$$
G_{i k \beta}^{j a}=\Lambda_{i k}^{j} \delta_{\beta}^{a} .
$$

Let us consider the case where

$$
t^{j a}(x, y)=u^{j}(x) v^{\alpha}(x, y), \quad v^{a^{\prime}}\left(x^{i^{\prime}}, y^{\beta^{\prime}}\right)=B_{\alpha}^{\alpha^{\prime}} v^{a}\left(x^{i}, y^{\beta}\right) .
$$

Further, we put

$$
\begin{aligned}
\dot{\nabla}_{i}\left(u^{j} v^{a}\right) & \stackrel{\text { df }}{=} v^{a} u_{; i}^{j}+u^{j} v_{; i}^{a}=v^{a}\left(u_{, i}^{j}+\Lambda_{i k}^{j} u^{k}\right)+u^{j}\left(v_{, i}^{a}+\tilde{\Gamma}_{i \beta}^{a} v^{\beta}\right) \\
& =\left(u^{j} v^{a}\right)_{, i}+\Lambda_{i k}^{j} u^{k} v^{a}+\tilde{\Gamma}_{i \beta}^{a} u^{j} v^{\beta} .
\end{aligned}
$$


Leibnitz's formula (1.9) will be satisfied when the space $X^{n}$ is a space $L^{n}$, and besides that there must be defined a product object $\tilde{\Gamma}_{i \beta}^{a}$, which we call the product linear connexion. In our subsequent considerations we shall show how to express the product object $\tilde{\Gamma}_{i \theta}^{a}$ by a linear connexion $\Gamma_{\gamma \beta}^{\alpha}$ (if $\bar{Y}^{\bar{n}}$ is $L^{\vec{n}}$ with a linear connexion $\Gamma_{\gamma \beta}^{a}$ ).

From (1.9) we obtain

$$
G_{i k \beta}^{j a}=\Lambda_{i k}^{j} \delta_{\beta}^{a}+\tilde{\Gamma}_{i \beta}^{a} \delta_{k}^{j} .
$$

We assume that relation (1.1.0) is fulfilled for an arbitrary product tensor (1.1); thus

$$
\dot{\nabla}_{i} t^{j a}=t_{, i}^{j a}+\Lambda_{i k}^{j} t^{k \alpha}+\tilde{\Gamma}_{i \beta}^{a} t^{j \beta} \text {. }
$$

Making use of (1.6) and (1.10) it is easy to verify that the law of transformation of the product linear connexion has the form

$$
\tilde{\Gamma}_{i^{\prime} \beta^{\prime}}^{a^{\prime}}=A_{i^{\prime}}^{i} B_{a}^{a^{\prime}} B_{\beta^{\prime}}^{\beta}, \tilde{\Gamma}_{i \beta}^{a}-A_{i^{\prime}}^{i} B_{\beta^{\prime}}^{a} B_{a i}^{a^{\prime}} .
$$

This connexion is a product tensor when $B_{a i}^{a^{\prime}}=0$. One can easily verify that the product linear connexion is the product geometric object, which we omit.

Remark 1.1. Let

$$
\Gamma_{k a}^{\gamma} \stackrel{\text { df }}{=} W_{k}^{\beta} \Gamma_{\beta a}^{\gamma},
$$

where $\Gamma_{a \beta}^{\gamma}$ is a linear connexion on the manifold $Y^{\bar{n}}$, and $W_{k}^{\theta}$ is a priori arbitrary product object. But from (1.12), (1.13) and from the law of transformation of the linear connexion [2], [5] we get

$$
\begin{gathered}
\tilde{\Gamma}_{k^{\prime} \alpha^{\prime}}^{\gamma^{\prime}}=W_{k^{\prime}}^{\beta^{\prime}} \Gamma_{\beta^{\prime} \alpha^{\prime}}^{\gamma^{\prime}}=W_{k^{\prime}}^{\beta^{\prime}}\left(B_{\gamma}^{\gamma^{\prime}} B_{a^{\prime}}^{a} B_{\beta^{\prime}}^{\beta} \Gamma_{\beta a}^{\gamma}-B_{a^{\prime}}^{a} B_{\beta^{\prime}}^{\beta} B_{\beta_{a}}^{\gamma^{\prime}}\right), \\
A_{k^{\prime}}^{k} B_{a^{\prime}}^{a} B_{\gamma}^{\gamma^{\prime}} \Gamma_{k a}^{\gamma}-A_{k^{\prime}}^{k} B_{a^{\prime}}^{a} B_{a k}^{\gamma^{\prime}}=W_{k^{\prime}}^{\beta^{\prime}}\left(B_{\gamma}^{\gamma^{\prime}} B_{a^{\prime}}^{a} B_{\beta^{\prime}}^{\beta} \Gamma_{\beta a}^{\gamma}-B_{\beta^{\prime}}^{\beta} B_{a^{\prime}}^{a} B_{\beta a}^{\gamma^{\prime}}\right) .
\end{gathered}
$$

The last relation gives

$$
W_{k^{\prime}}^{\beta^{\prime}}=A_{k^{\prime}}^{k} B_{\beta}^{\beta^{\prime}} W_{k}^{\beta}, \quad W_{k^{\prime}}^{\beta^{\prime}} B_{\beta^{\prime}}^{\beta} B_{\beta a}^{\gamma^{\prime}}=A_{k^{\prime}}^{k} B_{a k}^{\gamma^{\prime}} .
$$

If $B_{a \dot{r}}^{\gamma^{\prime}}=0$, then the product object $W_{k}^{\beta}$ is an arbitrary product tensor. The derivative

$$
B_{a k}^{\gamma^{\prime}}=\frac{\partial^{2} y^{\gamma^{\prime}}}{\partial x^{k} \partial y^{a}}
$$

is non-zero when $y^{a}=y^{a}\left(x^{i}\right)$. Then the relation

$$
B_{a k}^{\gamma^{\prime}}=B_{a \beta}^{\gamma^{\prime}} y_{, k}^{\theta},
$$

where $y_{, k}^{\beta}=\frac{\partial y^{\beta}}{\partial x^{k}}$ is fulfilled. We eventually get

$$
W_{k^{\prime}}^{\beta^{\prime}}=A_{k^{\prime}}^{k} B_{\beta}^{\beta^{\prime}} y_{, k}^{\theta} \text {, }
$$


whence

$$
W_{k}^{\beta}=y_{, k}^{\beta} \text {. }
$$

In Section 2 of [6] the formulae for the covariant derivative of $(H, \bar{H})$-product tensors $\omega_{e}^{r}, \omega_{r e}$ have been given. Now let us consider the case where these $(H, \bar{H})$-product tensors are simply product tensors. We take two such product tensors: $T_{a}^{j}, V_{j a}$. From formulae (2.27), (2.28) of [6] we get

$$
\begin{aligned}
\dot{\nabla}_{i} V_{j a} & =V_{j a, i}+\tilde{G}_{i j a}^{k \beta} \nabla_{k \beta}, \\
\dot{\nabla}_{i} T_{a}^{j} & =T_{a, i}^{j}+\bar{G}_{i k a}^{j \beta} T_{\beta}^{k},
\end{aligned}
$$

respectively. Reasoning in an analogical way as in deriving relation (1.10), we obtain

$$
\tilde{G}_{i j a}^{k \beta}=h_{i j}^{k} \delta_{a}^{B}+\tilde{h}_{i \alpha}^{\beta} \delta_{j}^{k} .
$$

Imposing additionally the condition

we get

$$
t^{j a} \nabla_{j a}=\sigma, \quad \sigma_{; i}=\sigma_{, i}=\nabla_{j a} \dot{D}_{i} t^{j a}+t^{j a} \dot{\nabla}_{i} V_{j a},
$$

$$
h_{i j}^{k}=-\Lambda_{i j}^{k}, \quad \tilde{h}_{i \alpha}^{\beta}=-\tilde{\Gamma}_{i \alpha}^{\beta} \quad \tilde{G_{i k \beta}^{j a}}=-G_{i k \beta}^{j a} ;
$$

thus

$$
\dot{\nabla}_{i} V_{j a}=\nabla_{j a, i}-\Lambda_{i j}^{k} V_{k a}-\tilde{\Gamma}_{i a}^{\beta} V_{j \beta}
$$

From our previous considerations it is evident that formulea (1.11), (1.19) for the covariant derivative of product tensors are formally (mnemotechnically) the same as those for the covariant derivative of single tensors [2], [5]. Thus relation (1.16) has the form

$$
\dot{\nabla}_{i} T_{a}^{j}=T_{a, i}^{j} \dashv-\Lambda_{i k}^{j} T_{a}^{k}-\tilde{\Gamma}_{i \alpha}^{\beta} T_{\beta}^{j} .
$$

Remark 1.2. One can assume relation (1.7) as the definition of the covariant derivative of product tensors. Then, of course, manifolds $X^{n}, Y^{\vec{n}}$ must be spaces with linear connexions, because, e.g.,

$$
\dot{\nabla}_{\beta} T_{a}^{m}=T_{a ; \beta}^{m}=T_{a, \beta}^{m}-\Gamma_{a \beta}^{\gamma} T_{\gamma}^{m} .
$$

Ericksen [1] assumes formulae (1.7), (1.21) ex definitio, and calls them partial covariant derivatives.

2. Now we deal with the case where there exists a mapping of class $C^{1}, h: X^{n} \rightarrow Y^{\bar{n}}$, which we write down in coordinate systems in the following way:

$$
y^{a}=y^{a}\left(x^{j}\right), \quad j=1, \ldots, n, a=1, \ldots, \bar{n}
$$


We can interpret relations (2.1) in one more way; namely, they can be understood as the equations of a hypersurface $X^{n}$ imbedded in a space $Y^{\bar{n}}$. In the latter case we must have $n \leqslant \bar{n}-1$ [2], [5].

Let manifolds $X^{n}, \bar{Y}^{\bar{n}}$ have linear connexions $\Lambda_{k i}^{m}, \Gamma_{\beta \gamma}^{\alpha}$, respectively. We know that the covariant derivative $\dot{\nabla}_{i}$ of the product tensor $t^{\text {ma }}$ has the form (see formula (1.11))

$$
\dot{\nabla}_{i} t^{j a}=t_{, i}^{j \alpha}+\Lambda_{i k}^{j} t^{k a}+\tilde{\Gamma}_{i \beta}^{a} t^{j \beta} .
$$

Let us take in (2.2), instead of the partial derivative $t_{, i}^{j \alpha}$, the total derivative

$$
\frac{d t^{j a}\left(x^{k}, y^{\theta}\left(x^{m}\right)\right)}{d x^{i}}=\frac{\partial t^{j a}}{\partial x^{i}}+\frac{\partial t^{j a}}{\partial y^{\beta}} \frac{\partial y^{\beta}}{\partial x^{i}}=t_{, i}^{j a}+t_{, \beta}^{j a} y_{, i}^{\beta},
$$

where $y_{, i}^{\beta}=\frac{\partial y^{\beta}}{\partial x^{i}}$.

Further, we assume that relation (1.13) is fulfilled, where $W_{k}^{\beta}=y_{, k}^{\beta}$ (see Remark 1.1), i.e.

$$
\tilde{\Gamma_{i \beta}^{a}}=y_{, i}^{\gamma} \Gamma_{\gamma \beta}^{a} .
$$

Taking account of (2.3), (2.4) in (2.2), we get

$$
\begin{aligned}
t_{\| i}^{j a} & =t_{, i}^{j a}+\Lambda_{i k}^{j} t^{k a}+\left(t_{, \beta}^{j a}+\Gamma_{\beta \gamma}^{a} t^{j \nu}\right) y_{, i}^{\beta} \\
& =t_{; i}^{j a}+t_{; \beta}^{j a} y_{, i}^{\beta} .
\end{aligned}
$$

We have assumed the symbol $\|$ in (2.5). to denote the covariant derivative of product tensors for which we take the total derivative instead of the partial derivative.

It is evident that expression (2.5) presents a product tensor, because both the covariant derivatives $t_{; i}^{j a}, t_{; \beta}^{j a}$ and the partial derivatives $y_{, i}^{\beta}$ are product tensors.

Ericksen [1] calls the covariant derivative (2.5) the total covariant derivative, but he assumes this relation ex definitio, as a generalization of the total derivative of the scalar $\sigma\left(x^{i} y^{a}\left(x^{j}\right)\right)$.

Let us assume that $n=\bar{n}$, and let a mapping $h: X^{n} \rightarrow Y^{n}$, be a homeomorphism of class $C^{1}$. Transvection $x^{i},=\partial x^{i} / \partial y^{\gamma}$ with both sides of (2.5) and taking into account that

$$
y_{, i}^{\beta} x_{, \gamma}^{i}=\delta_{\gamma}^{\beta}
$$

we obtain

$$
t_{\| i}^{j a} x_{, \gamma}^{i}=t_{; \gamma}^{j a}+t_{; i}^{j a} x_{, \gamma}^{i}=t_{\| \gamma}^{j a} .
$$

In an analogical way we get

$$
t_{\| i}^{j a}=t_{\| \beta}^{j a} y_{, i}^{\beta} .
$$


Final remarks. The formulae for the covariant derivative of product tensors are also valid for double and connecting tensors; we mean here relations (1.11), (1.19), (1.20).

Examples of the applications of the covariant derivative of product tensors in the theory of elasticity have been given in [4].

If relations (2.1) present the equations of a hypersurface $X^{n}$ imbedded in a space $\bar{Y}^{\bar{n}}, n \leqslant \bar{n}-1$, then Goląb [2] denotes the connecting tensor $y_{, i}^{a}$ by $C_{i}^{a}$, and Schouten [5] by $B_{i}^{a}$. It is easy to verify that relations (10.26) given in [2] on p. 303, are a special form of our formula (1.20). Namely, it is sufficient to take into account (2.4) in (1.20) and to assume $\Lambda_{i k}^{j}$ as the linear connexion of the imbedded space $X^{n}$. In this case relation (2.5) is also satisfied.

Schouten [5], p. 285-287, introduces the Cartesian product of spaces $L^{n}$, $L^{n-m}$ (with linear connexions). The Cartesian product of such spaces is the space $L^{n}$ for which all components of the linear connexion with two indices of a different kind vanish. In our considerations it would mean that the product linear connexion $\tilde{\Gamma}_{i \beta}^{\alpha}$ vanishes (see (1.11), (1.19), (1.20)).

\section{References}

[1] J. L. Ericksen, Double tensor fields, Encyclopedia of Physics III/1, Springer, 1960.

[2] S. Gołąb, Rachunek tensorowy, Warszawa 1966.

[3] M. Kucharzewski, Objekte des Kartesischen Produktes zweier Mannigfaltig. keiten, Ann. Polon. Math. 20 (1968), p. 215 - 221.

[4] W. Noll, The equations of finite elasticity. Application of non-linear partial differontial equations, Math. Phys., Providence, R. I. Amer. Math. Soc. (1966), p. $93 \cdot 101$.

[5] J. A. Schouten, Ricci-calculs, Springer, 1954.

[6] J. J. Telega, On covariant derivative of product scalars and $(H, \bar{H})$-product tensors, Prace Mat. U.S. (in print).

[7] - Pewne typy liniowych, jednorodnych obiektów iloczynowych (podwbjnych, rozdwojonych) klasy pierwszej, Z.N. Polit. Śl., Mat.-Fiz. (in print). 